University of Wollongong

Research Online

Faculty of Business - Papers (Archive)

Faculty of Business and Law

$1-1-2014$

Reassessing Edgeworth's conjecture when population dynamics is stochastic

Simone Marsiglio

University of Wollongong, simonem@uow.edu.au

Follow this and additional works at: https://ro.uow.edu.au/buspapers

Part of the Business Commons

Research Online is the open access institutional repository for the University of Wollongong. For further information contact the UOW Library: research-pubs@uow.edu.au 


\title{
Reassessing Edgeworth's conjecture when population dynamics is stochastic
}

\begin{abstract}
We analyze the implications of different welfare criteria on economic and population growth in the case of stochastic population change. Edgeworth (1925) argues that total utilitarianism leads to a lower economic performance and a larger population size than average utilitarianism. Following works show that while his intuition holds in a static framework, the result is unclear in a dynamic setting of endogenous growth. We show that if population dynamics is stochastic, Edgeworth's conjecture may or may not hold. In particular, which utilitarian criterion implies larger economic and population growth rates depends on the value of the inverse of the intertemporal elasticity of substitution, the features of the random process driving demographic change and the magnitude of the (linear) dilution coefficient. We also characterize the size of the range of parameter values supporting Edgeworth's conjecture; such a range is wide if the dilution parameter is large enough while it is particularly narrow (but still non-empty) if the parameter is small.
\end{abstract}

\section{Keywords}

dynamics, population, when, reassessing, conjecture, edgeworth, stochastic

Disciplines

Business

\section{Publication Details}

Marsiglio, S. (2014). Reassessing Edgeworth's conjecture when population dynamics is stochastic. Journal of Macroeconomics, 42 130-140. 


\title{
Reassessing Edgeworth's Conjecture when Population Dynamics is Stochastic
}

\author{
Simone Marsiglio* \\ Forthcoming in the Journal of Macroeconomics
}

\begin{abstract}
We analyze the implications of different welfare criteria on economic and population growth in the case of stochastic population change. Edgeworth (1925) argues that total utilitarianism leads to a lower economic performance and a larger population size than average utilitarianism. Following works show that while his intuition holds in a static framework, the result is unclear in a dynamic setting of endogenous growth. We show that if population dynamics is stochastic, Edgeworth's conjecture may or may not hold. In particular, which utilitarian criterion implies larger economic and population growth rates depends on the value of the inverse of the intertemporal elasticity of substitution, the features of the random process driving demographic change and the magnitude of the (linear) dilution coefficient. We also characterize the size of the range of parameter values supporting Edgeworth's conjecture; such a range is wide if the dilution parameter is large enough while it is particularly narrow (but still non-empty) if the parameter is small.
\end{abstract}

Keywords: Edgeworth's Conjecture, Stochastic Population, Economic Growth, Welfare Criteria JEL Classification: O40, O41, J13

\section{Introduction}

Different ways of defining the social welfare function have been proposed in the economic literature. Probably the most diffused approaches (at least in macroeconomic theory) are average and total (or classical) utilitarianism, respectively based on the so-called Millian and Benthamite criterion. The former says that social welfare coincides with per-capita utility while the latter that social welfare is the sum of individual utility across the population (per-capita utility multiplied by the population size, if agents are homogeneous). Several studies try to figure out how the way we define social welfare is going to affect the final outcome of the model, and in particular which criterion leads to faster economic and demographic growth. The issue is particularly popular in the optimal population size ${ }^{1}$ literature, which wonders what is the optimal, or the

* James Cook University, School of Business, PO Box 6811, Cairns QLD 4870, Australia. Contact: simone.marsiglio@jcu.edu.au. I am grateful to Pavel Brunovsky, Alberto Bucci, Davide La Torre and Fabio Privileggi for insightful discussions. I also thank participants at workshops and conferences held in Marseille, Strasbourg, Alessandria and Bratislava in 2012 for their helpful comments and suggestions. I wish also to thank the Editor, Ping Wang, and two anonymous referees for their constructive comments on earlier drafts of the paper. All remaining errors and omissions are my own sole responsibility.

${ }^{1}$ Parfit (1984) with his famous 'repugnant conclusion' raises a strong critique to total utilitarianism, by stating that: "For any possible population of at least ten billion people, all with a very high quality of life, there must be some much larger imaginable population whose existence, if other things are equal, would be better even though its members have lives that are barely worth living". This means that under total utilitarianism, it might be optimal to choose increasing population size even if this is associated with an 'embarassingly low' average standard of living. Such a critique is relevant in a static framework where resources are fixed, but not in a dynamic framework of endogenous growth where average welfare keeps rising over time (see Razin and Yuen, 1995; and Boucekkine and Fabbri, 2013). Recently, Boucekkine and Fabbri (2013) stress this result by showing that Parfit's repugnant conclusion may occur only along a 'degenerate' balanced growth path, where per-capita consumption decreases over time. 
most advantageous, number of lives in a population under given circumstances; the typical view on such a question is that the optimal population has to ensure the largest social welfare, and thus how social welfare is defined has clear implications on the possible answer.

Edgeworth (1925) is the first to conjecture that the Benthamite criterion leads to a larger population size and a lower economic performance. Following studies (as Nerlove et al., 1982 and 1985) support his intuition: in a purely static context, the Benthamite principle implies larger population size and lower standards of living. The argument is simple: total utilitarianism allows for a substitutability between number of people and their individual wellbeing; since marginal utility is decreasing, to a large extent it is convenient to grow a larger population even if this may subtract resources from individual consumption. More recent analysis shows that in a dynamic framework of endogenous growth, the result is not so obvious: some works (Palivos and Yip, 1993; and Boucekkine and Fabbri, 2013) show that the Benthamite criterion leads to smaller population size and higher economic growth, while others (Razin and Yuen, 1995) demonstrate exactly the opposite 2 . Palivos and Yip (1993) analyze a canonical AK-type growth model where agents' utility depends both on consumption and fertility, and capital dilutes linearly with population growth. Boucekkine and Fabbri (2013) consider a similar model where fertility does not affect utility but is related to capital accumulation by a nonlinear dilution function. Razin and Yuen (1995) deal with a different framework where fertility affects (linearly) human capital accumulation and (nonlinearly) population growth, in order to stress the role of the quality-quantity trade-off of children. In Palivos and Yip (1993), the direct effect of population growth on welfare is twofold since individuals gain utility from the number of children they have (i.e., fertility is an argument of the utility function) and this determines the size of the household (i.e., population size multiplies utility); in both Razin and Yuen (1995) and Boucekkine and Fabbri (2013), population growth has a direct effect on welfare only through the latter channel.

In this paper we try to shed some light on the utilitarian trade-off in dynamic models of endogenous growth, and in particular we aim at reassessing Edgeworth's conjecture by extending the analysis to a stochastic context, since uncertainty about future population is an important aspect that has received only little attention so far. Demographic shocks, which may represent population booms, wars, catastrophic events, changes in immigration, family or health policies, are thought to have serious effects of macroeconomic variables, especially on growth rates (Robertson, 2002). Even the United Nations (UN), which regularly publish the "World population prospects" (WPP), probably the projections most widely used by international organizations, governments and researchers for planning and monitoring activities, recognize the importance of demographic uncertainty for future world development. Indeed, since the first publication of the WPP three different (deterministic) scenario projections ("low", "medium" and "high") have been presented in order to emphasize the potential implications of uncertainty on world population; as a recognition of the dramatically increasing role of uncertainty in the current world of fixed resources with rising and aging population, in its latest versions the UN have recently introduced a formal stochastic component $\mathrm{H}^{3}$ in their predictions (UN, 2013). Despite this compelling need to take into account demographic uncertainty, apart from some sporadic attempt the economic literature has remained silent on the topic, and no study

\footnotetext{
${ }^{2}$ Other related works include Strulik (2005), Bucci (2008), Marsiglio and La Torre (2012a) and Boucekkine et al. (2013). However, their goals are quite different from ours, since they consider the implications of exogenous population growth on endogenous R\&D (Strulik, 2005; and Bucci, 2008) and multi-sector growth (Marsiglio and La Torre, 2012a; Boucekkine et al., 2013) models.

${ }^{3}$ From the 2010 revision, the UN Population Division has introduced probabilistic methods for projecting total fertility and thus total population growth. Specifically, the process used rely on Bayesian hierarchical models, and allow to distinguish countries according to the stage of the demographic transition they are currently facing (see Hafner and Mayer-Foulkes, 2013, for a recent empirical analysis of the different phases of the demographic transition experienced by developed and less developed countries). Further details about the estimation and forecast techniques employed in the last 2012 revision of the WWP can be found in Raftery et al. (2013).

${ }^{4}$ On the theoretical side, see Robertson (2002), and Marsiglio and La Torre (2012b), who use a comparative statics exercise and a stochastic optimization problem, respectively, to analyze a two-sector endogenous growth model subject to random demographic shocks (see also Smith, 2007, who focuses on a one-sector growth model). On the empirical side, see Azomahou et
} 
has tried to relate the issue to the optimal population size problem.

To the best of our knowledge, all the existing works, both in a static and dynamic framework, focus on the deterministic version of the problem, and uncertainty about population change is not taken into account. In this paper we try to fill this gap by adopting a simple approach allowing for analytical results. Specifically, we assume, as standard in growth theory, that the instantaneous utility function depends only on consumption (as in Razin and Yuen, 1995; Boucekkine and Fabbri, 2013) and we consider two different specifications of the model. In section 2, we first analyze a simple framework where population dynamics is completely exogenous (as in Strulik, 2005; Bucci, 2008; and Marsiglio and La Torre, 2012a) in order to obtain an obvious benchmark for the following analysis; this assumption thus allows us to evaluate only the implications of different utilitarian approaches on economic performance. We show that which utilitarian criterion leads to faster growth depends on two factors: the magnitude of the inverse of the intertemporal elasticity of substitution and the features of the (completely exogenous) random process driving population dynamics. The simplification is removed in section 3, where we endogenize the trend of population growth by assuming that capital accumulation is nonlinearly affected by demographic growth (as in Marsiglio, 2011; Boikos et al., 2013; and Boucekkine and Fabbri, 2013). Thus, since population change is endogenously determined, we can evaluate also the effects of different utilitarian approaches on population size. We show that, also in this case, the magnitude of the intertemporal elasticity of substitution and the characteristics of the random process driving population dynamics affect the utilitarian trade-off; however, a third factor, that is the magnitude of the (linear) dilution coefficient, determines the overall result. We show that Edgeworth's conjecture may hold if certain parameter conditions are met, and through numerical simulations we assess how large is the range of parameter values allowing for such an outcome to occur. Section 4 as usual contains concluding remarks and proposes directions for future research.

\section{Exogenous Population Growth}

The model is a standard Ramsey-type (1928) model of optimal growth where the social planner seeks to maximize the welfare of the society subject to the economic and demographic constraints, choosing the consumption level, $c(t)$, of the representative agent. The welfare is the infinite discounted sum of the product of the instantaneous utility function (assumed to be iso-elastic, $u(c(t))=\frac{c(t)^{1-\theta}}{1-\theta}$, where $\theta>0, \neq 1$ is the inverse of the intertemporal elasticity of substitution) and the population size, $N(t)$, weighted by the planner's degree of altruism. Demographic dynamics is stochastic and is driven by a geometric Brownian motion, as in Smith (2007): $d N(t)=n N(t) d t+N(t) \sigma d W(t)$, where $n>0$ is the drift and $\sigma \geq 0$ the variance parameter, while $d W(t)$ is the increment of a Wiener process. The economic constraint is given by the law of motion of capita $5^{5} K(t)$, which depends on the difference between net (of depreciation, $\delta$ ) production (assumed to be linear in capital, with $A$ being a scale parameter representing the total factor productivity) and consumption: $\dot{K}(t)=A K(t)-\delta K(t)-N(t) c(t)$.

Given the initial conditions of capital and population, $K(0)>0$ and $N(0)>0$, respectively, the planner problem can be summarized as:

$$
\begin{array}{ll}
\max _{c(t)} & W=E_{0}\left[\int_{0}^{\infty} \frac{c(t)^{1-\theta}}{1-\theta} N(t)^{1-\epsilon} e^{-\rho t} d t\right] \\
\text { s.t. } & \dot{K}(t)=A K(t)-\delta K(t)-N(t) c(t) \\
& d N(t)=n N(t) d t+N(t) \sigma d W(t) .
\end{array}
$$

The term $1-\epsilon$, where $\epsilon \in[0,1]$, represents the degree of intratemporal altruism and depends on the number of individuals determining the same generation (the weight assigned by the planner to the size of future

al. (2009) which assesses the spatial impact of demographic shocks on economic growth.

${ }^{5}$ The notion of capital we adopt in the paper needs to be taken in a wide sense, encompassing physical (Palivos and Yip, 1993), human (Razin and Yuen, 1995) or even natural (Marsiglio, 2011) capital. 
generations). This is different from the intertemporal altruism, represented by the rate of time preference, $\rho$, which depends on the discount rate applied to future population wellbeing 6 . Note that the value of $\epsilon$ drives the assumption we are making about the social welfare function (see Palivos and Yip, 1993; and Razin and Yuen, 1995). In fact, if $\epsilon=0(\epsilon=1)$ we are adopting a total (average) utilitarianism approach, and the welfare function is of the Benthamite (Millian) type.

Define $J(K(t), N(t))$ as the maximum expected value associated with the stochastic optimization problem described above. The Hamilton-Jacobi-Bellman (HJB) equation is:

$\rho J(K(t), N(t))=\max _{c(t)}\left\{\frac{c(t)^{1-\theta}}{1-\theta} N(t)^{1-\epsilon}+J_{K}[A K(t)-\delta K(t)-N(t) c(t)]+J_{N} n N(t)+\frac{1}{2} \sigma^{2} J_{N N} N(t)^{2}\right\}$,

where subscripts denote partial derivatives of $J$ with respect to the relevant variables of interest. Dropping the $t$ s for clarity, differentiating (4) with respect to consumption gives:

$$
c=J_{K}^{-\frac{1}{\theta}} N^{-\frac{\epsilon}{\theta}}
$$

which substituted back into (4) yields:

$$
0=\left(\frac{\theta}{1-\theta}\right) J_{K}^{-\frac{1-\theta}{\theta}} N^{\frac{\theta-\epsilon}{\theta}}-\rho J+A J_{K} K-\delta J_{K} K+n J_{N} N+\frac{1}{2} \sigma^{2} J_{N N} N^{2} .
$$

By applying the guess and verify method to the previous equation, it is possible to show that a closed form solution for the problem exists, provided that a certain parameter condition is met.

Proposition 1. Assume $A<\delta+\frac{\rho-(\theta-\epsilon)\left[n-\frac{1}{2} \sigma^{2}(1-\theta+\epsilon)\right]}{1-\theta}$; then the HJB equation 6) has a solution given by:

$$
J(K(t), N(t))=\Psi K(t)^{1-\theta} N(t)^{\theta-\epsilon},
$$

where:

$$
\Psi=\frac{\theta^{\theta}}{(1-\theta)\left\{\rho-(1-\theta)(A-\delta)-(\theta-\epsilon)\left[n-\frac{1}{2} \sigma^{2}(1-\theta+\epsilon)\right]\right\}^{\theta}} .
$$

Moreover, the optimal path of consumption and capital are respectively:

$$
\begin{aligned}
c(t) & =\Omega k(t), \\
K(t) & =K(0) e^{(A-\delta-\Omega) t},
\end{aligned}
$$

where $\Omega=\frac{\rho-(1-\theta)(A-\delta)-(\theta-\epsilon)\left[n-\frac{1}{2} \sigma^{2}(1-\theta+\epsilon)\right]}{\theta}$ and $k(t)=\frac{K(t)}{N(t)}$.

The assumption in Proposition 1 is needed in order to ensure that the objective function is bounded, similarly to what happens in deterministic AK models to rule out explosive behavior. Equation (8) says that at equilibrium there exists a linear relationship between the optimal level of consumption and capital. This result is standard both in this stochastic optimization setup (Smith, 2007; Bucci et al., 2011), and in the deterministic AK-type context (Rebelo, 1991; Boucekkine and Fabbri, 2013). Note that the value function in Proposition 1 is consistent with that derived by Boucekkine and Fabbri (2013). As in Smith (2007), substituting the consumption function into equation (2) and applying Ito's lemma yields the equilibrium growth rate of (per-capita) capital, $\gamma(t)$ :

$$
\gamma(t) \equiv \frac{d k(t)}{k(t)}=\left[\frac{A-\delta-\rho-\epsilon n+\frac{1}{2} \sigma^{2}\left[\theta+\epsilon+(\theta-\epsilon)^{2}\right]}{\theta}\right] d t-\sigma d W(t) .
$$

\footnotetext{
${ }^{6}$ Both the intratemporal and intertemporal altruism parameters are related to future generations; the former measures the weight assigned by the planner to the size of future generations while the latter the weight assigned to the wellbeing of future generations.
} 
Since in such a framework the growth rate of consumption and output is equal to the growth rate of capital, the previous expression represents also the economic growth rate; see equation (8). Note that this is constant, apart from the randomness due to population change; as for any AK model, (the trend of) economic growth turns out to be positive if the total factor productivity is large enough (but not too large). We can now analyze the relationship between economic growth and the altruism parameter. By differantiating (10) with respect to $\epsilon$, we get:

$$
\frac{\partial \gamma(t)}{\partial \epsilon}=\frac{1}{\theta}\left[\sigma^{2}\left(\frac{1}{2}+\epsilon-\theta\right)-n\right] d t .
$$

The previous expression shows that the impact of altruism on economic growth is determined by two distinct factors. The latter term, $-\frac{n}{\theta}$, represents what we may label 'deterministic effect', since it is directly related to the trend of population growth; such a factor is consistent with previous works (Palivos and Yip, 1993; Marsiglio and La Torre, 2012a), and it says that as long as population growth (its trend) is positive increases in the altruism parameter will decrease economic growth. The former term, $\frac{\sigma^{2}}{\theta}\left(\frac{1}{2}+\epsilon-\theta\right)$, represents what we may refer to as 'uncertainty effect', since it mainly depends upon the volatility of population growth; to the best of our knowledge such an element has never been analyzed before, and it shows that increases in volatility will alternatively increase or decrease economic growth according to some parameter conditions. We note that the previous derivative will be positive if the uncertainty effect is positive and larger than the deterministic effect, that is $\frac{1}{2}+\epsilon-\theta>\frac{n}{\sigma^{2}}$, otherwise it will be negative. This condition depends only on the features of the stochastic process driving population dynamics, that is on the ratio between its trend and variance. It is interesting to compare this result with the deterministic case, $\sigma=0$. In fact, if population growth is completely deterministic, the uncertainty effect vanishes and the (negative) deterministic effect dominates; this implies that the previous condition is never met, and therefore the relationship between growth and altruism is monotonically decreasing. This confirms that in a deterministic setup of endogenous growth total utilitarianism leads to a higher growth rate (Palivos and Yip, 1993; Boucekkine and Fabbri, 2013; and Marsiglio and La Torre, 2012a), and thus no space is left for Edgeworth's conjecture. We have just shown the following result:

Proposition 2. If population dynamics is stochastic, there exists a non-monotonic relationship between economic growth and the altruism parameter. If instead population growth is deterministic, such a relationship is monotonically decreasing.

More interesting is understanding which criterion leads to a larger growth rate when demography is random. By setting $\epsilon=0$ and $\epsilon=1$ in equation 10 , we obtain respectively the Benthamite, $\gamma^{B}(t)$, and the Millian, $\gamma^{M}(t)$, growth rates:

$$
\begin{aligned}
& \gamma^{B}(t)=\left[\frac{A-\delta-\rho+\frac{\theta}{2} \sigma^{2}(1+\theta)}{\theta}\right] d t-\sigma d W(t) \\
& \gamma^{M}(t)=\left[\frac{A-\delta-\rho-n+\frac{1}{2} \sigma^{2}\left[\theta+1+(\theta-1)^{2}\right]}{\theta}\right] d t-\sigma d W(t) .
\end{aligned}
$$

If the deterministic result that total utilitarianism leads to faster growth is confirmed also in the case of stochastic population change, then we will have $\gamma^{B}(t)>\gamma^{M}(t)$. By comparison, we can see that in order for this result to occur, we need that $\frac{n}{\sigma^{2}}>1-\theta$, and in such a case, the economic growth rate under total utilitarianism will be larger. Two elements affect whether this condition holds or not: the value of the inverse of the intertemporal elasticity of substitution and the features of the random process driving population change. If $\theta>1$ then automatically $\gamma^{B}(t)>\gamma^{M}(t)$. If $\theta<1$ then $\gamma^{B}(t)>\gamma^{M}(t)$ only if the population trend to variance ratio is large enough; thus, in the case such a ratio is small the Benthamite criterion will lead to a smaller economic growth rate, as conjectured by Edgeworth (1925). We can summarize this as follows: 
Proposition 3. If $\theta>1$, then the Benthamite criterion leads to a larger economic growth than the Millian principle. If $\theta<1$, then the Benthamite criterion leads to a faster (slower) growth than the Millian principle whenever $\frac{n}{\sigma^{2}}>1-\theta$ (whenever $\frac{n}{\sigma^{2}}<1-\theta$ ).

Proposition 3 shows that both a range of parameters under which Edgeworth's conjecture may and may not hold in a stochastic dynamic framework exist. If $\theta>1$ there are no possibilities for the Millian growth rate to be larger than the Benthamite one; if $\theta<1$, this could happen 7 . The intuition behind this type of relationship between the inverse of the intertemporal elasticity of substitution and Edgeworth's conjecture is related to the interplay between the consumption smoothing attitude, economic and population growth. Whenever the propensity to smooth consumption out is small $\left(\theta<1-\frac{n}{\sigma^{2}}\right)$ events far in the future affect the consumption growth pattern strongly; thus a reduction in the weight of future generations (higher $\epsilon)$ might more than offset the (potentially) negative uncertainty-induced effects related to demographic change (measured by $\frac{n}{\sigma^{2}}$ ) increasing economic growth, and as a result average utilitarianism might lead to higher economic performance lending support to Edgeworth's claim 8 . Whenever the propensity to smooth consumption out is large $\left(\theta>1-\frac{n}{\sigma^{2}}\right)$ such a counterbalancing effect is not possible thus there is no chance for average utilitarianism to increase economic growth.

Since Edgeworth's hypothesis might occur only if certain conditions are met, it may be worthwhile to test how large is the set of parameters supporting Edgeworth's intuition. Figure 1 shows how the condition identified in Proposition 3 varies for different values of $\theta$ and $\sigma$, for fixed value of $n$; the shaded area represents the set of parameter values where $\gamma^{B}(t)<\gamma^{M}(t)$ while the unshaded area that where $\gamma^{B}(t) \geq \gamma^{M}(t)$. We set $n=0.02$ as to represent the growth rate of population in industrialized countries, and we let $\theta$ and $\sigma$ vary ( $\theta$ varies between 0 and 1 which is the relevant interval; $\sigma$ between 0 and 2 for the sake of simplicity). The figure shows that for any value of $\theta<1$, there exists a range of values for $\sigma$ confirming Edgeworth's hypothesis; however, in order to ensure this, the variance of population change should be not too small. By changing the value of $n$ it is possible to show that this conclusion is robust: in order for the Millian principle to imply a better economic performance than the Benthamite criterion, the variance parameter has to be large enough 9

Intuitively, these results are related to the source of non-monotonicity in the growth and altruism rela-

${ }^{7}$ Thus the unity represents a critical value for the inverse of the intertemporal elasticity of substitution. We are aware of the fact that the $\theta>1$ case represents the empirically most plausible situation while the $\theta<1$ case is empirically less relevant. However, since the estimation of the size of $\theta$ is still controversial we do not enter the debate on the magnitude of this parameter here, and we consider all the possible values it might take in our analysis. Indeed, even if most of the empirical studies show that $\theta$ is larger than one (Hall, 1988), some others show that it may even be smaller (Hansen and Singleton, 1983). Also in theoretical works, the benchmark value of this parameter is thought to be greater than one (Barro and Sala-i-Martin, 2004); however in several works in the context of endogenous fertility (Marsiglio, 2011) or closed-form solutions of optimal growth models (Smith, 2006), it is often set less than one as well. Moreover, on a technical note, if $\theta>1$ the problems turns out to be not well posed, since such a case violates one of the two boundary conditions, $J(0, N)=0$ and $J(k, 0)=0$, which should supplement the HJB equation; notice that whenever $\theta>1$ the former condition is not met (since $\lim _{k \rightarrow 0} \Psi k^{1-\theta} N^{1-\epsilon}=\infty$ ) while whenever $\theta<1$ it is. We should thus restrict our analysis to the $\theta<1$ case (which is exactly the parametric restriction supporting our main results; see Proposition 3); however, as a matter of analytical scrupulousness, even the case $\theta>1$ is discussed in the paper.

${ }^{8}$ Note that the population trend to variance ratio measures the impact of demographic change on economic growth, thus when $\frac{n}{\sigma^{2}}$ increases (decreases) the propensity to smooth consumption out needs to get smaller (larger) in order for such a result to hold. This explains why in the deterministic case Edgeworth's conjecture cannot hold (see Proposition 2): when $\sigma=0$, the intertemporal elasticity of substitution should get so small to become (unrealistically) negative in order for the Millian to exceed the Benthamite growth rate.

${ }^{9}$ To the best of our knowledge, clear empirical estimates of the variance of population change are not present in the literature, and this is related to the widely diffused view that it may be changing over time and from country to country. Indeed, in population projections based upon Bayesian methods, as those developed by the UN, the variance parameter is generally assumed to be time dependent and related to the level of population growth, in order to distinguish between short run and long run trends which are associated to different phases of the demographic transition (UN, 2013). Recent results show that demographic uncertainty may lead to substantial differences in the estimates derived from a deterministic rather than a stochastic model (Raftery et al., 2009; UN, 2013). This suggests that the variance of population change may be large enough, lending support to our conclusion that the Millian growth rate might be larger than the Benthamite growth rate. 


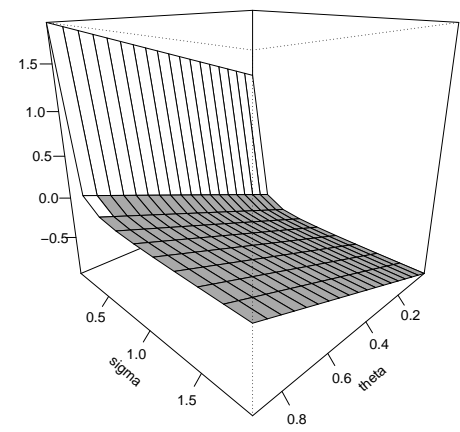

Figure 1: Range of values (shaded area) supporting Edgeworth's conjecture; trend of population growth, $n$, set equal to 0.02 .

tionship. As previously discussed, $\frac{\partial \gamma(t)}{\partial \epsilon}$ will be positive only if the uncertainty effect is strictly positive and larger than the deterministic effect. The above condition requiring the variance not to be small, basically ensures that the former effect is large enough to more than offset the latter (shaded area in the Figure 1). This means that the range of values supporting Edgeworth's hypothesis implies that the uncertainty effect dominates. In the alternative scenario, where the variance is small, as in the deterministic framework, the deterministic effect dominates and no space is left for Edgeworts' conjecture.

By comparing Proposition 2 and 3 , it is clear that as long as population dynamics is deterministic, we know a priori what is the effect of adopting total rather than average altruism on economic growth (and that Edgeworth's hypothesis cannot occur); once uncertainty is taken into account, the result is no longer obvious. Indeed, according to the features of the random process and the magnitude of the intertemporal elasticity of substitution, a faster growth may be ensured by total or average altruism.

\section{Endogenous Population Growth}

Edgeworth's (1925) hypothesis concerns the effect of alternative utilitarian criteria on both the economic and population growth rates. Since we considered population dynamics to be completely exogenous in the previous section, we could just assess the former portion of his conjecture. In order to assess also the latter part, we need to endegenize the trend of population growth. In doing so and in order to maintain the same utility function traditionally considered in growth theory (depending only on consumption), we follow Boucekkine and Fabbri (2013) and assume that the relationship between population growth and capital accumulation is nonlinear $[10$. This is to capture the fact that an inverted-U relationship between demographic and economic growth may exist (see Boucekkine et al., 2002), as supported by empirical evidence (see Kelley and Schmidt, 1995; and recently, Boikos et al., 2013). We thus modify the law of motion of capital as follows: $\dot{K}(t)=A K(t)-\delta K(t)-N(t) c(t)-\phi(n(t)) K(t)$, where $\phi(n(t))=a_{1} n(t)^{2}+a_{2} n(t)+a_{3}$ is a nonlinear dilution function, with $a_{1}>0, a_{2} \in(-\infty, 1)$ and $a_{3}>0$. Note that $a_{2}<1$ ensures that economic performance is positively (negatively) affected by population change for smaller (larger) values of the demographic growth

\footnotetext{
${ }^{10}$ Boucekkine and Fabbri (2013) allow for the linkage between demographic growth and capital accumulation to take any form, specifying then such a relationship as being either linear or nonlinear. The nonlinear situation is the most interesting case which we analyze in detail in this section. The linear case is qualitatively not different from what we analyzed in the previous section, since when the dilution function is linear optimality will be given by corner solutions, thus the optimal population growth rate will be independent of the altruism parameter allowing to assess only one part of Edgeworth's conjecture (exactly as in our section 2 .
} 
rate 11 . Now that the trend of population growth is endogenously determined by economic agents, the law of motion of population becomes: $d N(t)=n(t) N(t) d t+N(t) \sigma d W(t)$. Population change is still driven by a Brownian motion but its trend may change over time since it is a control variable. We shall refer to the trend of population growth as the fertility rate for the sake of simplicity, even if no fertility decisions are directly analyzed in the mode $\sqrt{12}$

The stochastic optimization problem now reads as:

$$
\begin{aligned}
\max _{c(t), n(t)} & W=E\left[\int_{0}^{\infty} \frac{c(t)^{1-\theta}}{1-\theta} N(t)^{1-\epsilon} e^{-\rho t} d t\right] \\
\text { s.t. } & \dot{K}(t)=A K(t)-\delta K(t)-N(t) c(t)-\left[a_{1} n(t)^{2}+a_{2} n(t)+a_{3}\right] K_{t} \\
& d N(t)=n(t) N(t) d t+N(t) \sigma d W(t) .
\end{aligned}
$$

Similarly to what we showed in the exogenous population case, it is possible to obtain a closed form solution for the HJB equation associated to the previous problem.

Proposition 4. Assume $A<\delta+a_{3}+\frac{\rho+\frac{1}{2} \sigma^{2}(\theta-\epsilon)(1-\theta+\epsilon)-\frac{1}{2}\left[\theta-\epsilon-a_{2}(1-\theta)(1-2 \theta)\right] \bar{n}}{1-\theta}$; then the value function associated to the problem (11), (12) and (13) is:

$$
J(K(t), N(t))=\Psi K(t)^{1-\theta} N(t)^{\theta-\epsilon},
$$

where:

$$
\Psi=\frac{\theta^{\theta}}{(1-\theta)\left\{\rho-(1-\theta)\left(A-\delta-a_{3}\right)-\frac{1}{2} \sigma^{2}(\theta-\epsilon)(\theta-\epsilon-1)-\frac{1}{2}\left[\theta-\epsilon-a_{2}(1-\theta)\right] \bar{n}\right\}^{\theta}} .
$$

Moreover, the optimal path of consumption and fertility are respectively:

$$
\begin{aligned}
c(t) & =\Omega k(t) \\
n(t) & =\bar{n}=\frac{1}{2 a_{1}}\left(\frac{\theta-\epsilon}{1-\theta}-a_{2}\right),
\end{aligned}
$$

where $\Omega=\frac{\rho-(1-\theta)\left(A-\delta-a_{3}\right)+\frac{1}{2} \sigma^{2}(\theta-\epsilon)(1-\theta+\epsilon)-\frac{1}{2}\left[\theta-\epsilon-a_{2}(1-\theta)\right] \bar{n}}{\theta}$.

The result is pretty similar to what we obtained in the case of exogenous population change: consumption is proportional to capital and the condition needed to rule out explosive paths is slightly different since now the trend of population growth is endogenously determined and we have introduced a nonlinear dilution term. Equation (16) shows that the fertility rate is constant and independent of the shocks affecting population dynamics; moreover it results to be positive if $a_{2}<\frac{\theta-\epsilon}{1-\theta}$, since $a_{2}$ can take any sign. Note that the fertility rate coincides with that derived by Boucekkine and Fabbri (2013) in a similar deterministic setup. Even if the randomness of population change is completely irrelevant for the determination of the optimal fertility rate, it affects the equilibrium economic growth rate which is given by the following expression:

$$
\gamma(t)=\left[\frac{A-\rho-\delta-a_{3}+\frac{1}{2} \sigma^{2}\left[\theta+\epsilon+(\theta-\epsilon)^{2}\right]+\frac{2 \theta \epsilon-\theta-\epsilon-a_{2}(1-\theta)}{2(1-\theta)} \bar{n}}{\theta}\right] d t-\sigma d W(t)
$$

\footnotetext{
${ }^{11}$ The inverted-U relationship between demographic and economic growth holds for per-capita variables. Abstracting for a moment from the randomness of population change (i.e., setting $\sigma=0$ ), the law of motion of per capita capital reads as: $\dot{k}(t)=A k(t)-\delta k(t)-c(t)-\left[a_{1} n(t)^{2}+\left(a_{2}-1\right) n(t)+a_{3}\right] k(t)$. If $a_{2}<1$, then population pressure becomes a burden for economic growth when $n(t)>\frac{1-a_{2}}{2 a_{1}}$, as suggested by empirical evidence (see Kelley and Schmidt, 1995; Boikos et al., 2013). Boikos et al. (2013) conclude their empirical investigation of the relationship between birth rates, human capital and economic growth, by stating that "[...] at low levels of the birth rate higher birth rates can surely have a positive growth-effect and [...] the main policy initiatives here should concentrate on easing the opportunity cost of having children. On the other hand, at high levels of the birth rate, a reduction in these rates can definitely contribute to boost per capita human capital accumulation and economic growth".

${ }^{12}$ According to Nerlove and Raut's (1997) classification, our model should be labeled as a model of endogenous population change, rather than a model of endogenous fertility, since 'no decision-making mechanism is presupposed'.
} 
The comments highlighted in the exogenous population case still apply in this framework, and the trend of the economic growth rate is positive if a certain condition on the total factor productivity holds. By differentiating (17) with respect to $\epsilon$ we can still identify the two terms discussed in the previous section:

$$
\frac{\partial \gamma(t)}{\partial \epsilon}=\frac{1}{\theta}\left\{\sigma^{2}\left(\frac{1}{2}+\epsilon-\theta\right)-\left(\frac{\frac{1}{2}-\theta}{1-\theta}\right) \bar{n}+\left[\frac{2 \theta \epsilon-\theta-\epsilon-a_{2}(1-\theta)}{2(1-\theta)}\right] \frac{\partial \bar{n}}{\partial \epsilon}\right\} d t
$$

The first term, $\frac{\sigma^{2}}{\theta}\left(\frac{1}{2}+\epsilon-\theta\right)$ represents the uncertainty effect, and it perfectly coincides with that identified in the case of exogenous population growth. The second term, $-\frac{\frac{1}{2}-\theta}{\theta(1-\theta)} \bar{n}$, represents the deterministic effect, which now does not have necessarily to be negative (because of the introduction of a nonlinear dilution function), and its sign depends on a certain parameter condition. However, since now the trend of population growth is optimally determined, there is a third term, $\left[\frac{2 \theta \epsilon-\theta-\epsilon-a_{2}(1-\theta)}{2 \theta(1-\theta)}\right] \frac{\partial \bar{n}}{\partial \epsilon}$, which we may label 'fertility effect', measuring the impact of altruism on the endogenous fertility rate; also the direction of such an effect depends on a certain parametric restriction. For the same reasoning developed in the previous section, the growth rate is a non-monotonic function of the altruism parameter, however, also in the case of deterministic population change $(\sigma=0)$ the sign of the previous derivative is not clearly defined, because both the sign of the deterministic and the fertility effect are not unambiguously determined (and they both depend on the size of $\theta$ ). About the relationship between fertility and altruism, since $\frac{\partial \bar{n}}{\partial \epsilon}=-\frac{1}{2 a_{1}(1-\theta)}$, it is straightforward to see that this uniquely depends on the size of the inverse of the intertemporal elasticity of substitution: if $\theta>1$ fertility increases with altruism (total utilitarianism leads to smaller population size, $\bar{n}^{B}<\bar{n}^{M}$ ) while if $\theta<1$ fertility decreases as altruism increases (total utilitarianism implies larger population size, $\bar{n}^{B}>\bar{n}^{M}$ ). Note that only the latter case is consistent with Edgeworth's intuition.

In order to fully assess the utilitarian trade-off, we need to compare the Benthamite and the Millian economic growth rates, which read as follows:

$$
\begin{aligned}
& \gamma^{B}(t)=\left[\frac{\left.A-\rho-\delta-a_{3}+\frac{1}{2} \theta \sigma^{2}(1+\theta)+\frac{a_{2}^{2}(1-\theta)^{2}-\theta^{2}}{4 a_{1}(1-\theta)^{2}}\right] d t-\sigma d W(t)}{\theta}\right] \\
& \gamma^{M}(t)=\left[\frac{A-\rho-\delta-a_{3}+\frac{1}{2} \sigma^{2}\left[\theta+1+(\theta-1)^{2}\right]+\frac{(1-2 \theta)+2 a_{2}(1-\theta)^{2}+a_{2}^{2}(1-\theta)^{2}+\theta^{2}}{4 a_{1}(1-\theta)^{2}}}{\theta}\right] d t-\sigma d W(t) .
\end{aligned}
$$

By comparing such two rates, we can see that $\gamma^{B}(t)>\gamma^{M}(t)$ only if a condition similar to that we obtained in the exogenous population case holds, namely $\theta-1>\frac{\mu}{\sigma^{2}}$, where $\mu \equiv \frac{1-2(1-\theta)\left(\theta-a_{2}(1-\theta)\right)}{4 a_{1}(1-\theta)^{2}}$. By comparing these two conditions, we can see that while in the previous section the magnitude of the intertemporal elasticity of substitution was compared to the (exogenous) trend to variance ratio, now it is compared with a similar ratio whose numerator is endogenously determined by the choice of the fertility rate; such a ratio still depends on the exogenous features of the random process driving population change (i.e., its variance). Which criterion leads to a faster growth depends on the sign of $\mu$ (whether the linear dilution parameter, $a_{2}$, is larger or smaller than a certain threshold, $\left.\alpha \equiv \frac{2 \theta(1-\theta)-1}{2(1-\theta)^{2}}\right)$, on the characteristics of the process driving population change (the magnitude of $\sigma$ ) and on the size of the inverse of the intertemporal elasticity of substitution (whether $\theta$ is larger or smaller than 1 ). It is thus possible to conclude the following:

Proposition 5. Assume $\theta>1$; if $a_{2} \leq \alpha$ then the Benthamite criterion leads to a larger economic growth than the Millian principle while if $a_{2}>\alpha$ this is true only if $\theta-1>\frac{\mu}{\sigma^{2}}$. Assume now $\theta<1$; if $a_{2}<\alpha$ then the Benthamite criterion leads to a larger economic growth than the Millian principle whenever $\theta-1>\frac{\mu}{\sigma^{2}}$ while if $a_{2} \geq \alpha$ this never holds.

Proposition 5 distinguishes among alternative scenarios for different parameter values. Equivalently, it could be stated in terms of the values of the linear dilution parameter. In the case $a_{2}<\alpha$, two outcomes are 
possible: if $\theta>1$ the Benthamite criterion will lead to a larger economic growth than the Millian principle, while if $\theta<1$ this will be the case only if also the condition $\theta-1>\frac{\mu}{\sigma^{2}}$ is met. In the case $a_{2}=\alpha$; the Benthamite criterion will lead to a larger (smaller) economic growth than the Millian principle if $\theta>1$ (if $\theta<1$ ). In the last case, namely $a_{2}>\alpha$, the two possible outcomes are the following: if $\theta>1$ the Benthamite criterion will imply a larger economic growth than the Millian principle only if the condition $\theta-1>\frac{\mu}{\sigma^{2}}$ is met while if $\theta<1$ this will never be the case. If we compare Proposition 5 with Proposition 3 , we can see that the variety of possible scenarios is richer when the fertility rate is endogenously determined, since a further condition related to the magnitude of $a_{2}$ (due to the presence of the fertility effect), is needed to fully characterize the utilitarian trade-off.

Recalling that Edgeworth's conjecture states that total utilitarianism leads to a larger population size and a lower economic performance (that is, $\bar{n}^{B}>\bar{n}^{M}$ and $\gamma^{B}(t)<\gamma^{M}(t)$ ), we can now check whether any parameter restriction supporting the claim exists. The Benthamite fertility rate turns out to be larger only in the case $\theta<1$; for such a range of the inverse of the intertemporal elasticity of substitution ${ }^{13}$, the Benthamite growth rate results to be smaller if $a_{2} \geq \alpha$, or also in the case $a_{2}<\alpha$ if the further condition $\theta-1<\frac{\mu}{\sigma^{2}}$ is satisfied (see Proposition 5). This means that Edgeworth's hypothesis can hold for any possible value of $a_{2}$. We have just shown the following result:

Proposition 6. Assume $\theta<1$; then Edgeworth's conjecture will hold for these two alternative parameter specifications: (i) $a_{2} \geq \alpha$; or (ii) $a_{2}<\alpha$ and $\theta-1<\frac{\mu}{\sigma^{2}}$.

An implication of Proposition 6 is that in the deterministic case $(\sigma=0)$, there is space for Edgewroth's intuition only if $a_{2}$ is sufficiently large (the case (i)), and this is related to the non-monotonic relationship between demographic and economic growth; as $a_{2}$ gets smaller, the randomness of population dynamics is needed to allow for Edgeworth's hypothesis to hold. Proposition 6 also confirms our previous finding that Edgeworth's conjecture is not compatible with values of the inverse of intertemporal elasticity of substitution larger than unity. In order to test whether the restrictions required by Proposition 6 are likely to occur for given parameter values, we can assess it graphically by plotting the two cases (i) and (ii) for different values of $\theta$ and $a_{2}$. Since from the previous section, we saw that the variance of the population process has to be large enough in order to support Edgeworth's claim, we set $\sigma=0.5$; we also set $a_{1}=1$ for the sake of simplicity. By changing the value of $\sigma$ and $a_{1}$, it is possible to show that the following conclusions are robust and the qualitative results do not differ substantially.

Figure 2 shows that the range of values of $\theta$ and $a_{2}$ allowing for the restriction (i) to hold is quite wide (this is represented by the shaded area in the figure); the condition is not satisfied only if both the inverse of intertemporal elasticity of substitution and the linear dilution coefficient get too small. For any value of $\theta$ (and, equivalently, for any value of $a_{2}$ ) there exist a set of values of $a_{2}$ (of $\theta$ ), satisfying the condition, and thus confirming Edgeworth's conjecture.

Figure 3 shows the case (ii). 3(a) and 3(b) represent the first and second parameter restriction required by this second case, respectively; the shaded area show whenever the conditions $\theta-1<\frac{\mu}{\sigma^{2}}$ (panel (a)) and $a_{2}<\alpha$ (panel (b)) are satisfied while the unshaded area when they are not. The range of values of $\theta$ and $a_{2}$ where both the panels present shaded areas is that supporting Edgeworth's hypothesis. It should be clear that the shaded area in $3(\mathrm{~b})$ is larger than the unshaded area in $3(\mathrm{a})$, and this implies that such a range of values is a non-empty set; however, by excluding the values leading to a negative fertility rate (that is excluding the values of $a_{2}>-1$ ), this range is particularly narrow, since it requires both $\theta$ and $a_{2}$ to lie in tiny intervals 14 . By comparing Figures 2 and 3 , it is clear that Edgeworth's conjecture is more likely to occur in the case in which $a_{2} \geq \alpha$; however, this is possible even in the case $a_{2}<\alpha$.

\footnotetext{
${ }^{13}$ Note that exactly as in the previous section, Edgeworth's conjecture may be verified only in the case in which the inverse of the intertemporal elasticity of substitution is smaller than one. The intuition behind the relationship between $\theta$ and $\epsilon$ is similar to what discussed earlier related to the interplay between the consumption smoothing attitude and population growth (along with the associated uncertainty).

${ }^{14}$ This can be illustrated through a specific example. Suppose $a_{2}=-1.01$ and $\theta=0.5$ (with $\sigma=0.5$ and $a_{1}=1$ ); then
} 


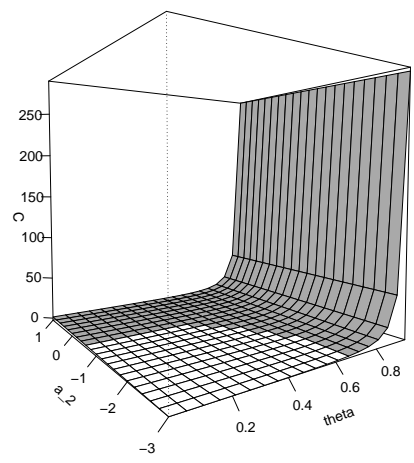

Figure 2: Range of values (shaded area) supporting Edgeworth's conjecture in the case (i); variance of population growth, $\sigma$, set equal to 0.5 , and $a_{1}$ equal to 1 .

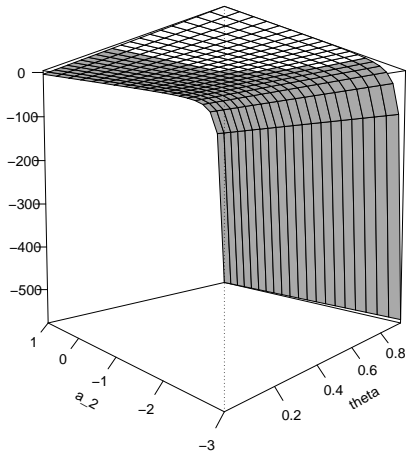

(a) Condition $\theta-1<\frac{\mu}{\sigma^{2}}$

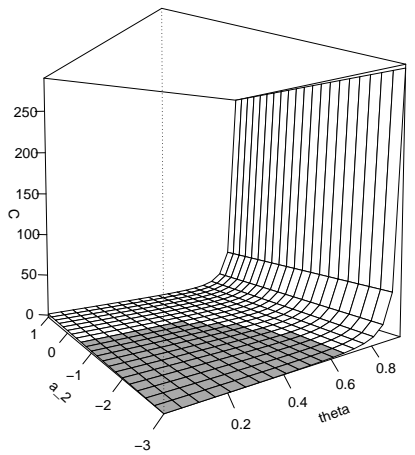

(b) Condition $a_{2}<\alpha$

Figure 3: Range of values supporting (shaded area simultaneously in 3(a) and 3(b) Edgeworth's conjecture in the case (ii); variance of population growth, $\sigma$, set equal to 0.5 , and $a_{1}$ equal to 1 .

In the previous section we discussed how Edgeworth's conjecture may hold only when the uncertainty effect is strictly positive and larger than the (negative) deterministic effect. In this case, where population growth is endogenously determined, since the deterministic effect may also be positive or null and also the fertility effect plays a crucial role in determining the sign of the growth and altruism relationship, disentangling the net impact of each of these three terms on economic performance is more complex. Intuitively, cases (i) and (ii) represent the possible scenarios in which the total effect on economic growth is positive. In particular, case (i) depicts the parameter values ensuring that the positive uncertainty and fertility effects are larger than the (positive or negative) deterministic effect, while case (ii) those associated to a negligible deterministic effect and a positive uncertainty (fertility) effect larger than the negative fertility (uncertainty) effect. Differently from the results in previous section, if the rate of population growth is endogenously determined, even when demographic change is completely deterministic, it will be possible for Edgeworth's conjecture to occur.

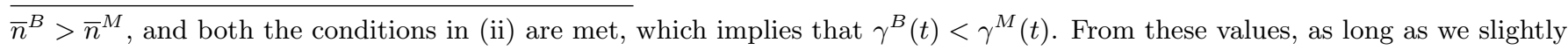
change $\theta$ or $a_{2}$ or both, the result is maintained; as the variation gets larger it disappears. 


\section{Conclusion}

This paper reassesses Edgeworth's (1925) conjecture, suggesting that the Benthamite criterion leads to a larger population size and a lower economic performance than the Millian criterion. In a static setup, it has been widely confirmed that Edgeworth's hypothesis is verified (Nerlove et al., 1982 and 1985). In a dynamic framework of endogenous growth, the result is unclear: some works (Palivos and Yip, 1993; Boucekkine and Fabbri, 2013) show this is no longer the case, while others (Razin and Yuen, 1995) still confirm his intuition. We show that if population dynamics is stochastic, total utilitarianism may lead to higher or lower economic and population growth than average utilitarianism. What will be the result is determined by three factors: the magnitude of the inverse of the intertemporal elasticity of substitution (whether it is larger or smaller than 1), the features of the random process driving population dynamics (whether its variance is large or small enough) and the size of the linear dilution coefficient (whether it is larger or smaller than $\alpha$ ). Therefore, in a dynamic framework with stochastic population change, Edgeworth's conjecture may hold if certain parameter conditions are met. In particular, for Edgeworth's conjecture to be verified, the inverse of the intertemporal elasticity of substitution has to be smaller than the unity, confirming that in the case it is larger than 1 there is no possibility for Edgeworth's conjecture to occur. By numerical simulations, we show that if the linear dilution coefficient is large enough there exists a wide range of parameters lending support to Edgeworth's hypothesis; if the linear dilution parameter is small such a range is particularly narrow, but it is still non-empty. Within this range of parameters, by moving from the Bethamite to the Millian social welfare function, even in a dynamic framework of endogenous growth, we could rise economic performance and at the same time lower demographic growth.

Note that our results supporting Edgeworth's (1925) intuition have been derived in two alternative settings. We have first considered the most common setup in the economic literature, namely (per capita) capital dilutes linearly with the exogenously given population growth rate; then, we moved to the most interesting case where capital dilutes nonlinearly with demographic change and the rate of population growth is endogenously determined. In the former case, the presence of randomness in the evolution of demography is essential in order for the Benthamite economic growth rate to be smaller than the Millian one; in the latter case randomness is not necessary for observing both a larger population size and a lower economic performance under the Millian principle, but it clearly rises its likelihood by increasing the impact of the uncertainty effect on the growth and altruism relationship. This confirms that the occurrence of Edgeworth's conjecture is a concrete possibility, which does not strictly depend upon our model specification. In line with the projections of the UN (2013), this also suggests that uncertainty about the evolution of future population is an important factor to take into account in order to clearly assess the welfare implications of different economic and demographic policies. This calls for the need of further (empirical and theoretical) works in order to improve our understanding of how uncertainty affects economic and demographic (and even environmental) development.

Our results represent only a preliminary attempt to analyze the economic and demographic implications of uncertainty, thus cannot be considered exhaustive. In particular, the framework we analyzed, namely a canonical endogenous growth (AK type) model in which instantaneous utility depends only upon consumption, is an immediate starting point for such considerations, however it has some limitations. First of all, such a specification suggests the existence of a complex relationship between the inverse of the intertemporal elasticity of substitution and population growth; very little is known on the nature of such an interaction, thus further analysis is needed in order to shed some light on the reciprocal implications between the elasticity of intertemporal substitution and demographic change. Moreover, the results derived in such a framework suggest that Edweworth's conjecture may hold only in the empirically less plausible case in which the inverse of the intertemporal elasticity of subsitution is smaller than one; such a conclusion is likely to be due to the fact that we cannot distinguish between the relative risk aversion and the intertemporal elasticity of substitution coefficients, thus it might be worthwhile to consider a recursive utility formulation 
as in Epaulard and Pommeret (2003) in order to allow for such a differentiation. Finally, our model's specification is extremely simplistic, thus it is natural to wonder whether and how our results would differ in a more complex setup, as a multi-sector model; specifically, extending the analysis in order to consider a two sector model driven by human capital accumulation as in Robertson (2002) or an endogenous R\&D model as in Bucci (2008) would allow to further clarify the mutual relationship between growth, (human) capital accumulation, demography and uncertainty. This is left for future studies.

\section{References}

1. Azomahou, T., Diebolt, C., Mishra, T. (2009). Spatial persistence of demographic shocks and economic growth, Journal of Macroeconomics 31, 98-127

2. Barro, R.J., Sala-i-Martin, X. (2004). Economic Growth (Cambridge, Massachusetts: MIT Press)

3. Boikos, S., Bucci, A., Stengos, T. (2013). Non-monotonicity of fertility in human capital accumulation and economic growth, Journal of Macroeconomics 38, 44-59

4. Boucekkine, R., de la Croix, D., Licandro, O. (2002). Human vintage capital, demographic trends and endogenous growth, Journal of Economic Theory 104, 340-375

5. Boucekkine, R., Fabbri, G. (2013). Assessing Parfit's repugnant conclusion within a canonical endogenous growth set-up, Journal of Population Economics 26, 751-767

6. Boucekkine, R., Martinez, B., Ruiz-Tamarit, J.R. (2013). Growth vs. level effect of population change on economic development: an inspection into human-capital-related mechanisms, Journal of Mathematical Economics 49, 312-334

7. Bucci, A. (2008). Population growth in a model of economic growth with human capital accumulation and horizontal R\&D, Journal of Macroeconomics 30, 1124-1147

8. Bucci, A., Colapinto, C., Forster, M., La Torre, D. (2011). Stochastic technology shocks in an extended UzawaLucas model: closed-form solution and long-run dynamics, Journal of Economics 103, 83-99

9. Epaulard, A., Pommeret, A. (2003). Recursive utility, endogenous growth, and the welfare cost of volatility, Review of Economic Dynamics 6, 672-684

10. Edgeworth, F.Y. (1925). Papers relating to political economy, Vol. III (Macmillan, London)

11. Hafner, K.A., Mayer-Foulkes, D. (2013). Fertility, economic growth, and human development casual determinants of the developed lifestyle, Journal of Macroeconomics 38, 107-120

12. Hall, R.E. (1988). Intertemporal substitution in consumption, Journal of Political Economy 96, 339357

13. Hansen, L.P., Singleton, K.J. (1983). Stochastic consumption, risk aversion, and the temporal behavior of asset returns, Journal of Political Economy 91, 249-265

14. Kelley, A.C., Schmidt, R.M. (1995). Population and economic growth correlations: the role of the components of demographic change, Demography 32, 543-555

15. Marsiglio, S. (2011). On the relationship between population change and sustainable development, Research in Economics 65, 353-364

16. Marsiglio, S., La Torre, D. (2012a). Population dynamics and utilitarian criteria in the Lucas-Uzawa model, Economic Modelling 29, 1197-1204

17. Marsiglio, S., La Torre, D. (2012b). A note on demographic shocks in a multi-sector growth model, Economics Bulletin 32, 2293-2299 
18. Nerlove, M., Raut, L.K. (1997). Growth models with endogenous population: a general framework, in (Mark, R.R., Oded, S., eds.), Handbook of Population and Family Economics, Elsevier, 1117-1174

19. Nerlove, M., Razin, A., Sadka, E. (1982). Population size and the social welfare function of Bentham and Mill, Economics Letters 10, 61-64

20. Nerlove, M., Razin, A., Sadka, E. (1985). Population size: individual choice and social optima, Quarterly Journal of Economics 100, 321-334

21. Palivos, T., Yip, C.K. (1993). Optimal population size and endogenous growth, Economics Letters 41, $107-110$

22. Parfit, D. (1984). Reasons and Persons (Oxford: Oxford University Press)

23. Raftery, A. E., Alkema, L., Gerland, P., Clark, S.J., Pelletier, F., Buettner, T., Sevcikova, H. (2009). White paper: probabilistic projections of the total fertility rate for all countries for the 2010 World Population Prospects, in United Nations expert group meeting on recent and future trends in fertility (United Nations Publication UN/POP/EGM-FERT/2009/16, Population Division, Department of Economic and Social Affairs, United Nations; retrieved from http://www.un.org/esa/population/meetings/EGM-Fertility2009/P16 Raftery.pdf)

24. Raftery, A.E., Alkema, L., Gerland, P. (2013). Bayesian population projections for the United Nations, Statistical Science, forthcoming

25. Ramsey, F. (1928). A mathematical theory of saving, Economic Journal 38, 543-559

26. Razin, A., Yuen, C.W. (1995). Utilitarian tradeoff between population growth and income growth, Journal of Population Economics 8, 81-87

27. Robertson, P.E. (2002). Demographic shocks and human capital accumulation in the Uzawa-Lucas model, Economics Letter 74, 151-156

28. Smith, W.T. (2006). A closed form solution to the Ramsey model, Contributions to Macroeconomics 6 , article 3

29. Smith, W.T. (2007). Inspecting the mechanism exactly: closed-form solution to a stochastic growth model, The B.E. Journal of Macroeconomics 7 (Contributions), Article 30

30. Strulik, H. (2005). The role of human capital and population growth in R\&D-based models of economic growth, Review of International Economics 13, 129-145

31. United Nations (2013). World Population Prospects: the 2012 Revision (United Nations Publication ST/ESA/SER.A/336, Population Division, Department of Economic and Social Affairs, United Nations; retrieved from http://esa.un.org/wpp/Documentation/publications.htm) 